\title{
DUST AND GAS: OVERVIEW
}

\author{
François Schweizer \\ Department of Terrestrial Magnetism \\ Carnegie Institution of Washington \\ 5241 Broad Branch Road, N.W. \\ Washington, DC 20015
}

\begin{abstract}
Progress in the 50 years since the discovery of ionized gas in NGC 1052 is reviewed. As discovery has proceeded from $\mathrm{H}$ II to $\mathrm{H}$ I and recently to $10^{7} \mathrm{~K}$ gas, the known amount of gas in ellipticals has increased dramatically: from $10^{3-6} M_{\odot}$ for $\mathrm{H}$ II, to $10^{5-9} M_{\odot}$ for $\mathrm{H}$ I in some $15 \%$ of E's, to $10^{9-10} M_{\odot}$ for the $\mathrm{X}$-ray emitting gas. Although a few ellipticals with dust have long been known, recent CCD surveys have revealed dust lanes in nearly half of all E's. The cooler gas - as traced by H I, warm H II, and dust - is generally distributed in the form of a disk that often shows an outer warp. To deduce the true shapes of ellipticals from the geometry and kinematics of such disks has proven a challenging task. There is mounting evidence that many disks consist of gas accreted by mass transfers and mergers, and that some of them may not yet have reached dynamical equilibrium. Some of the major unsolved problems concern the atmospheric balance between sources and sinks of gas, and the importance of cooling flows and galactic winds.
\end{abstract}

\section{OPTICAL OBSERVATIONS $1936-1976$}

If we disregard the discovery of the plasma jet of M87 by Curtis in 1918, which found its explanation only in the mid-1950's, then the discovery of dust and gas in ellipticals dates back 50 years. In describing the morphological class E, Hubble (1936) noted that "small patches of obscuring material are occasionally silhouetted against the luminous background, but otherwise these nebulae present no structural details." And while Mayall (1936) was testing a new spectrograph with good UV transmission for the Crossley telescope, he discovered the [O II] $\lambda 3727$ emission line in NGC 1052, an E known today as a prototypical LINER galaxy. Three years later, Mayall (1939) had first rough statistics about ionized gas in ellipticals, with one $\mathrm{E}$ out of 14 observed with the Crossley showing the [O II] line, and a second $\mathrm{E}$ with [O II] emission known from the Mt. Wilson work. By 1956, when the survey by Humason, Mayall, and Sandage was published, the detection rate for the [O II] line in ellipticals was $14 \%$ for Lick and $18 \%$ for Mt. Wilson.

With the HMS survey completed, the ground work was laid for the three most fundamental papers on gas in ellipticals to appear in the first 40 years. Sandage (1957), in studying the luminosity function and evolution of stars in globular clusters, noted that in $\mathrm{M} 3$ about $10^{5} M_{\odot}$ of gas must have been shed 
by stars evolving from the main sequence to white dwarfs. Drawing parallels to ellipticals with their deeper potential wells and better ability to retain gas, Sandage remarked: “... this gas has probably not escaped from these systems and makes up about one two-hundredth of the total mass. Could this be the origin of the [O II] emission at $\lambda 3727$ observed in E nebulae?" As we all know, much of the following 25 years has been spent searching for this mass of gas, and only recently have X-ray observations revealed what seems to be the bulk of the gas.

The other two fundamental papers of this period are by Minkowski \& Osterbrock (1959) and Osterbrock (1960) and deal with the physics of interstellar matter in ellipticals. Since these two papers are often overlooked in modern work, let me point out how surprisingly much was already known about the ionized gas from these early observations of two E's with the brightest emission lines: NGC 1052 and 4278. Minkowski and Osterbrock found that each galaxy has an extended region of ionized gas at its center, with a spectrum not unlike that of Orion. From the relative intensities of the two components of the [O II] doublet, it followed that $N_{e} \approx 10-300 \mathrm{~cm}^{-3}$ and that the total mass of ionized gas is $10^{4}-10^{6} M_{\odot}$ $\left(H_{0}=75\right)$. In both galaxies, the ionized gas seemed to be arranged in a flattened disk and to rotate rapidly. Toward the nucleus, the turbulent motions in the gas were seen to increase and reach values comparable to the velocity dispersion of the stars. Minkowski and Osterbrock pondered about the energy input and suggested that there may be enough UV radiation from blue horizontal-branch stars to explain the observed line emission. They also estimated that collisional excitation due to the turbulent gas motions could marginally supply the energy, the most likely continuous source being mass shed by stars on strongly radial orbits. They did worry, as we still do today, that the mass inflow into the nucleus would be excessive because of rapid cooling. Finally, they guessed that all ellipticals may possess gas, but that ionization conditions may differ.

\section{FINDING DUST AND GAS: 4 COMPONENTS}

In reviewing the developments of the past 10 years, it seems useful to distinguish four components of the interstellar medium: the dust, cool gas $\left(T<10^{2} \mathrm{~K}\right)$, warm gas $\left(\sim 10^{4} \mathrm{~K}\right)$, and hot gas $\left(>10^{6} \mathrm{~K}\right)$.

\subsection{Dust}

There has been a recent renaissance of interest in dust in ellipticals for two reasons: 1) Bertola and Galletta (1978) suggested that one can find the true shapes of some ellipticals from the orientation of their dust lanes; and 2) the advent of CCD detectors has made it easy to search for weaker dust features. Three techniques are now generally used to find dust from CCD images: digital unsharp masking (Schweizer \& Ford 1985), model reconstruction and masking (Lauer 1985), and color mapping (Carter et al. 1983; Sparks et al. 1985). Figure 1 shows dust and ripples in NGC 5018 revealed by unsharp masking. Several papers in this volume illustrate the other two techniques. All this recent activity has led to several major advances. First, a classification system for dust features introduced by Hawarden et al. (1981) has found widespread acceptance. Second, there now exist two main catalogs of dusty E's with about 150 entries combined (Ebneter \& Balick 1985; Sparks et al. 1985). And third, we now know that at least $25 \%-40 \%$ of field ellipticals have dust lanes detectable on modern photographs (Sadler \& Gerhard 

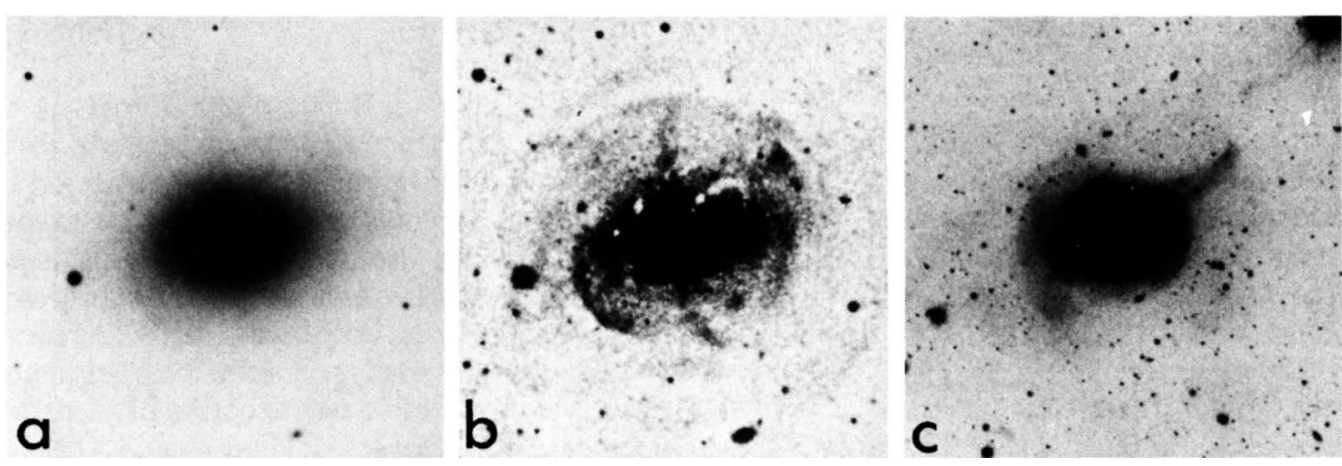

Figure 1. Fine structure in the E3 galaxy NGC 5018. (a) $3^{\prime} \times 3^{\prime}$ scan of a CTIO 4-m IIIa-J plate; $(b)$ unsharply masked image showing dust lanes, inner ripples, and plumes; $(c) 9^{\prime} \times 9^{\prime}$ scan at extremely high contrast showing outer ripples and tails (from Schweizer \& Ford 1985).

1985) and that dust masses in these galaxies are of order $10^{4}-10^{5} M_{\odot}$.

In the past, astronomers have worried that there may be much dust distributed uniformly throughout ellipticals. The main argument against this possibility has been, and still is, the small variation of color index among E's. Infrared observations from IRAS now confirm that there is very little warm dust in them (Rieke \& Lebofski 1986). It seems therefore likely that what little dust we see in obscuring patches is what dust there is. Yet major unsolved problems remain: Where does the dust come from, and why has it not yet been destroyed by the hot gas? A possible answer is that the dust is accreted together with cool gas and then survives in the gaseous disk.

\subsection{Cool Gas $\left(<10^{2} \mathrm{~K}\right)$ : H I}

In the early 1970's, the search for neutral hydrogen in E's turned out to be surprisingly difficult. The apparent lack of cool gas led to the hypothesis and wide acceptance of galactic winds that sweep E's clean of gas. But starting about 1976, $\mathrm{H}$ I was finally found in several ellipticals. Among the first to be detected were again NGC 4278 (Bottinelli \& Gouguenheim 1977; Gallagher et al. 1977) and NGC 1052 (Knapp et al. 1978; Fosbury et al. 1978). Figure 2 shows a modern VLA map of NGC 1052 made by van Gorkom et al. (1986). This map illustrates three points about H I distributions in E's: 1) H I can often be detected beyond the Holmberg radius and is roughly arranged in a disk or ring; 2) such disks often look perturbed and have occasionally appendices reminiscent of tidal tails; and 3) a potential donor galaxy can occasionally be seen nearby in the form of some gas-rich spiral.

From observations with sensitive modern detectors it appears that about $10 \%-15 \%$ of all E's have measurable amounts of H I (Knapp et al. 1985). Total $\mathrm{H}$ I masses range from a detection-limited $10^{5} M_{\odot}$ up to $\sim 5 \times 10^{9} M_{\odot}$. There also seems to be a clear environmental effect: E's in the Virgo and Pegasus clusters have very little $\mathrm{H}$ I, with upper limits of $5 \times 10^{7} M_{\odot}$ for Virgo (Kumar \& Thonnard 1983). Finally, molecules have not yet been discovered except in NGC 5128 (see review by Knapp in this volume). 

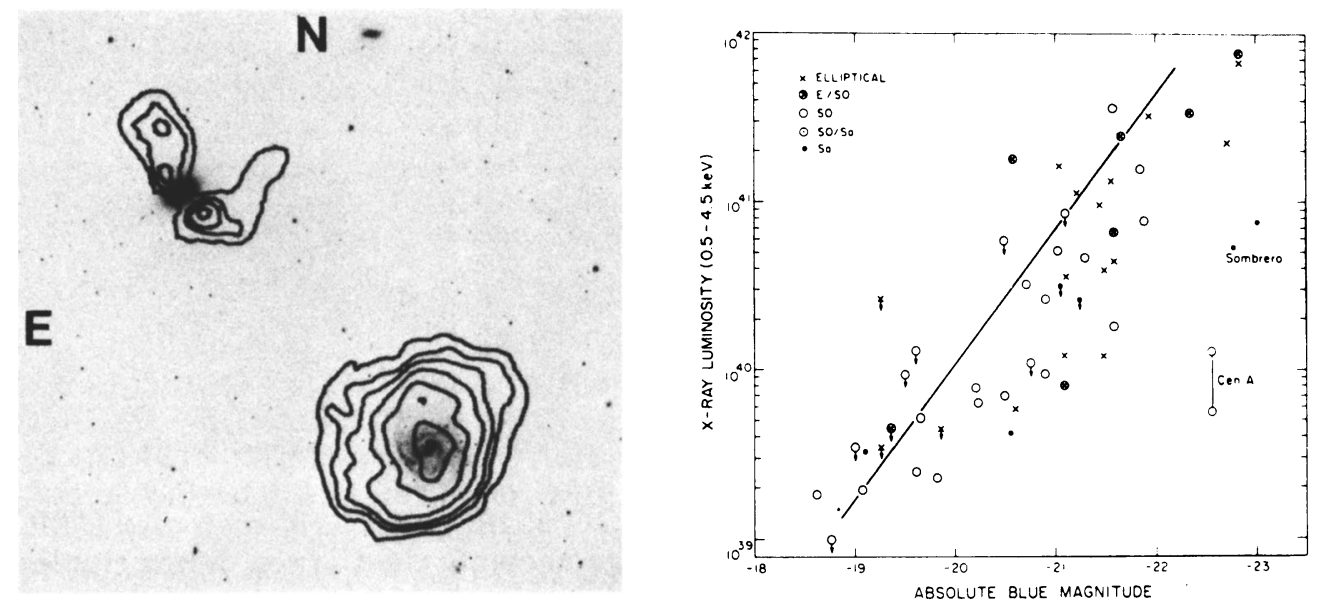

Figure 2. (left) VLA map of the H I distribution in NGC 1052 and in spiral companion NGC 1042 (from van Gorkom et al. 1986).

Figure 3. (right) X-ray luminosity of 55 early-type galaxies plotted against $M_{B}$. The line is not a best fit, but is the $L_{x}-M_{B}$ relation predicted if all X-ray emission arises from thermal bremsstrahlung of hot gas shed by evolving stars in $10^{10}$ years (from Forman et al. 1985).

\subsection{Warm Gas $\left(\sim 10^{4} \mathrm{~K}\right):$ H II}

Virtually all our knowledge about the warm, ionized gas in E's stems from optical spectroscopy. Some nice sample spectra of NGC 4278 and 5077 are shown by Demoulin-Ulrich et al. (1984); they are typical LINER spectra and are representative of most ionized regions observed in giant ellipticals. There exist now good spectra obtained with linear detectors for several hundred E's and S0's. Between one and two dozen of these galaxies have been observed in sufficient detail to determine the kinematics of their gas. As a result of improved techniques, the fraction of E's known to possess ionized gas has risen from the canonical $15 \%$ of HMS to $40 \%$ with [O II] $\lambda 3727$ detected on image-tube plates in the blue (Caldwell $1984 \mathrm{~b})$ and even to $55 \%-60 \%$ with the [N II] $\lambda 6584$ line detected on CCD frames in the red (Phillips et al. 1986). A significant part of the success of recent studies stems from a careful, digital subtraction of the galaxy continuum with its bumps and absorption lines.

These and other studies confirm many of Minkowski and Osterbrock's findings of a quarter century ago. Typical central electron densities are $N_{e} \approx 10^{3}$ $\mathrm{cm}^{-3}$ for $T \approx 10^{4} \mathrm{~K}$. Most ionized regions are small, of order $1 \mathrm{kpc}$ in diameter or less. Some, though, are considerably larger such as in NGC 5128 ( $D=8 \mathrm{kpc}$; Graham 1979) and NGC 1316 ( $D \approx 16 \mathrm{kpc}$; Schweizer 1980). The mass of ionized gas is of order $10^{3}-10^{6} M_{\odot}$, with Phillips et al. finding many E's near the lower limit. The ionized gas always seems to rotate much faster than the stars and is often arranged in an inclined disk. Near the nucleus, the turbulent velocity of the gas increases and reaches values between one-half and one time the velocity dispersion of the stars. The ionized gas, too, shows a strong environmental effect in the sense that nuclear emission is much reduced in dense clusters (Gisler 1978). Finally, a new result not known to Minkowski and Osterbrock is that the presence 
and strength of emission lines from ionized gas correlates well with the presence of dust and the X-ray luminosity of the galaxy.

\subsection{Hot Gas $\left(>10^{6} \mathrm{~K}\right)$ : X-ray Coronae}

The most exciting news of recent years has been the discovery that there does seem to be, after all, a lot of gas in E's and that it is in the form of hot coronae emitting X-ray radiation. As is well known, the EINSTEIN satellite with its highresolution imaging capability made it possible to identify extended X-ray emission sources with individual, massive E galaxies and to separate them from the general background emission in clusters. One of the first papers to report the detection of individual coronae pointed out already that there seemed to be nearly $10^{10} M_{\odot}$ of $10^{7} \mathrm{~K}$ gas in NGC 4406 (Forman et al. 1979).

Many EINSTEIN observations have been analyzed since. Forman, Jones, and Tucker (1985) and Trinchieri \& Fabbiano (1985) summarize observations of 55 early-type galaxies. They find that X-ray coronae around E's seem to be common if not ubiquitous, regardless of whether the E's are isolated or in clusters. In massive ellipticals, the coronae extend out to $100 \mathrm{kpc}$ radius and more, often well beyond the optical isophotal radius $R_{25}$. Particle energies are of order $1 \mathrm{keV}$, corresponding to $T \approx 10^{7} \mathrm{~K}$ and to r.m.s. velocities for protons of about $300 \mathrm{~km}$ $\mathrm{s}^{-1}$, or the same as the stellar velocity dispersions. The masses of hot gas seem to range between $5 \times 10^{8} M_{\odot}$ and $5 \times 10^{10} M_{\odot}$. The X-ray luminosity depends on nearly the square of the blue luminosity, suggesting that more luminous galaxies have more hot gas per unit blue luminosity (see Fig. 3). On the other hand, there are indications that in the least luminous galaxies a major fraction of the $\mathrm{X}$-ray emission may stem not from hot gas, but from low-mass binaries. Finally, if one assumes that the hot gas is isothermal (which is still under debate), one finds central particle densities of $\sim 0.01-0.1 \mathrm{~cm}^{-3}$, core radii for the gas distribution of $1-5 \mathrm{kpc}$, and short cooling times of order $10^{8} \mathrm{yr}$ near the center.

\section{CHEMICAL ABUNDANCES}

Any discussion of interstellar matter in ellipticals should include chemical abundances. Yet very little work has been done in this direction. In his 1960 paper, Osterbrock pointed out the similarity between the spectrum of the gas in NGC 4278 and that of the Orion nebula. He also pointed out the main difficulty in determining abundances, which is the lack of reliable temperatures and, therefore, the lack of information about the degree of ionization. The difficulty is that weak, temperature-sensitive lines, such as [O III] $\lambda 4363$, are buried in the continuum radiation of old stars, which itself is riddled with absorption lines. Osterbrock could only conclude that the logarithmic oxygen abundance is $[\mathrm{O} / \mathrm{H}] \approx 8.3$ or about $1 / 3$ solar if $T=10^{4} \mathrm{~K}$, but would be $[\mathrm{O} / \mathrm{H}] \approx 7.4$ or about $1 / 25$ solar if $T=2 \times 10^{4}$ $\mathrm{K}$. This basic difficulty remains today, as does the difficulty of choosing between photoionization and collisional ionization models.

On the empirical side, Phillips et al. (1986) have found a correlation between the line ratio $[\mathrm{N} \mathrm{II]} \lambda 6584 / \mathrm{H} \alpha$ and the blue luminosity of $\mathrm{E}$ and $\mathrm{S} 0$ galaxies, which may reflect abundance variations or changing ionization conditions. Ford \& Butcher (1979) have carried out a detailed analysis of the luminous filaments in M87 and find that their nitrogen and sulphur abundances exceed those in planetary nebulae of the Milky Way by factors $2-4$. Obviously, much more abundance work remains to be done. 


\section{SPATIAL DISTRIBUTION}

The four components of the interstellar matter in ellipticals appear in only two forms of distribution.

The hot gas of the X-ray coronae seems to have a space-filling, approximately spherical distribution. This has increasingly led to the use of the word "atmosphere" to describe it. The radial distribution can be represented by an isothermal distribution remarkably well over a range of $\sim 10^{3}$ in surface brightness (Forman et al. 1985).

The other three components - the dust, H I, and warm ionized gas - all seem to be arranged more or less in the form of a single disk. In different E's these disks are oriented at all conceivable angles with respect to the apparent axes of the projected light distribution. Hawarden et al. (1981) found that roughly one third of E's with dust lanes have them oriented along the apparent major axis, another third along the apparent minor axis, and the remaining third have them either at a skewed angle, or strongly distorted, or too irregular to classify. Warps in these disks are frequent and show up either in the dust lanes themselves, or as a progressive change in the rotation axis measured from the inner part of the disk to the outer part.

Young stars are occasionally associated with such disks, as in the well-known case of NGC 5128. Recently, the polar-ring galaxy AM 2020-5050 has been shown to be an elliptical surrounded by a blue ring of stars and a gaseous disk that stretches from the center to beyond this ring (Fig. 4a). Also, I have discovered a very faint, knotty ring of stars around IC 2006, a bona fide southern elliptical shown in Figure $4 \mathrm{~b}$, but have not yet been able to detect the ionized gas that presumably goes along with it. This is a good object to observe with a CCD spectrograph at $\mathrm{H} \alpha$ and might yield a reliable $M / L$ ratio in an elliptical far from the center.

\section{GAS KINEMATICS AND DYNAMICS}

The discovery of inclined and warped gas disks in ellipticals has opened up the possibility to learn about the shape of the potential, i.e., whether it is generally oblate, prolate, or triaxial. As a result, many theoretical and observational papers have been devoted to this subject during the past eight years. Some of them are discussed in more detail in the reviews by Bertola and Davies in this volume. Here, let me try to paint the broad picture, and point out where gains have been made and where more work is needed.

Most papers on the gas kinematics and dynamics can be assigned to one of four subject categories.

The earliest papers attempted to use existing observations to distinguish between oblate and prolate potentials that were assumed to be fixed in space. Bertola \& Galletta's (1978) discovery of a class of ellipticals with minor-axis dust lanes suggested that these galaxies would be good candidates for being prolate. Building on this and on Kahn \& Woltjer's (1959) notion of an inclined gaseous disk settling due to differential precession, Tohline, Simonson, \& Caldwell (1982) studied the existence of preferred planes into which the gas would settle in oblate 


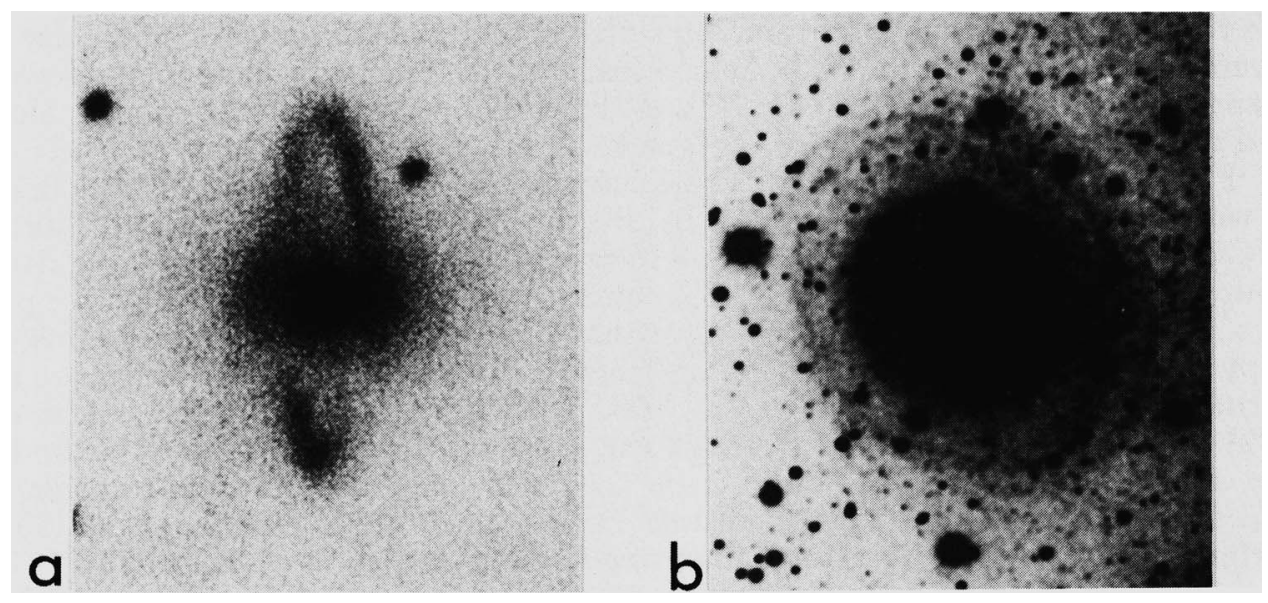

Figure 4. Young stars in outer rings. (a) Polar-ring elliptical AM 2020-5050 from Las Campanas 2.5-m plate (see Whitmore et al. in this volume); (b) IC 2006 (E1) and faint, knotty ring from CTIO $4-\mathrm{m}$ plate.

and prolate potentials; they proposed that of the 12 best observed E's, eight were prolate and only four were oblate. These were the golden days when the structure of ellipticals still looked seductively simple!

Yet, of course, Binney (1978) had already proposed that E's might be triaxial. Papers in the second group then took this refinement into consideration and studied gas orbits in triaxial potentials of fixed orientation. Much marvellous work was done here in Princeton by Martin Schwarzschild (1979) and his collaborators (e.g., Heiligman \& Schwarzschild 1979) who showed that in a fixed triaxial potential closed gas orbits do exist, that they are nearly elliptical, and that in any given potential these orbits may populate two preferred planes. Steiman-Cameron \& Durisen (1982) then demonstrated that polar orbits can be stable and can have unexpectedly large capture areas for gaseous infall. Yet the discovery of more types of possible gas orbits by Merritt \& de Zeeuw (1983) raised doubts that a one-to-one correspondance between the apparent orientation of dust lanes and the shape of the potential could be established. These authors emphasized that kinematical information would be needed to test the triaxiality of E's. A recent observational paper that finds evidence for triaxiality based on fixed-orientation models is the study of NGC 1052 by Davies \& Illingworth (1986).

In recent years, two major complications have arisen to make the analysis of observations of the gas kinematics more complex: For one, it was realized in the early 1980's that triaxial galaxies could be tumbling; and second, evidence started growing that the observed gas configurations may be transient.

Various people realized that a tumbling triaxial potential could give rise to inclined and warped gas disks due to the Coriolis force. Papers in the third group then analyzed the various possible configurations (Heisler, Merritt, and Schwarzschild 1982; Tohline \& Durisen 1982; Merritt \& de Zeeuw 1983, see their illustrated Table 1). Especially the beautiful paper by van Albada, Kotanyi, \& Schwarzschild (1982) made some precise predictions as to which way the stellar body should tumble if the gaseous disk is observed to rotate in a given direction and has a given warp. Namely, the tilt of the inclined gas disk should be such 
that, if you look down along the figure rotation axis, the gas orbits in a retrograde sense relative to the figure rotation. Sharples et al. (1983) applied this theory to the remarkable galaxy NGC 5363, which features an inner dust lane aligned with the apparent minor axis and an outer dust lane aligned with the major axis; they found a tumble period of close to $10^{9}$ years.

Finally, the fourth group of papers have emphasized evidence that inclined and warped gas disks may be transient. Beginning with Graham's (1979) study of NGC 5128, it has been found time and again that (1) the gas rotation axis is decoupled from the stellar rotation axis, and (2) the net angular momentum per unit mass of the gas is much larger than that of the stars. This has forced us to accept the notion that these disks do not consist of gas shed by stars, but have an external origin instead. In the case of NGC 5128 itself, where van Albada et al. (1982) made a specific prediction about the sense of rotation of the stellar body, three studies have now found, and agree among themselves, that the rotation is in the sense opposite from the prediction (Davies et al. 1984; Bertola et al. 1985; Wilkinson et al. 1986). Therefore, the warped gaseous disk of at least NGC 5128 is almost certainly a transient phenomenon, making it more difficult to establish the exact shape of the galaxy.

In studying the various observational papers on early-type galaxies with gas disks, I picked eight galaxies which were considered to be the best understood by the authors (with apologies to authors whose pet object I may have overlooked): NGC 1052 (E4), 1316 (D4), 4125 (E6), 5128 (E0p), 5266 (S0), 5363 (E5), 7070A (I0), and 7097 (E5). I find it impressive that four of these eight galaxies show direct evidence of having accreted disk material in the recent past: NGC 1316 has ripples or "shells" (Fig. 5a), as do NGC 5128 (Fig. 5b) and NGC 7070A (Sharples et al. 1983; Malin \& Carter 1983); and NGC 4125 has peculiar crossed streamers (Bertola et al. 1984). A fifth galaxy, NGC 7097, has stars and gas counter-rotating around the same axis (Caldwell et al. 1986), a configuration that can hardly be due to anything else but gas accretion from an external source. The remaining three objects each have a potential donor of gas nearby: NGC 5363 (Sharples et al. 1983) is only about three diameters away from the classical grand-design spiral NGC 5364 (Fig. 5c); NGC 1052 is close to the spiral NGC 1042 (Fig. 2); and even NGC 5266 (Caldwell 1984a) is within about seven diameters from the barred Sc galaxy NGC 5266A, though the latter's redshift is not yet known. The reason for suspecting relatively frequent mass transfers in close pairs is, of course, that the cross section for a mass transfer is of oider $10-10^{2}$ times greater than the cross section for a merger. An example of a mass transfer taking place is shown in Figure 5d.

In summary, it seems to me that the case for transient gas configurations as a general phenomenon is strong (for detailed theory, see Gunn 1979 and SteimanCameron \& Durisen 1987). We do seem to have found some galaxies where an oblate potential can nearly be excluded (e.g., Caldwell 1984a), and many recent papers find evidence for triaxiality (e.g., Bertola et al. 1985; Davies \& Illingworth 1986). Yet we should remain honest and admit that we are still unable to reliably determine the ratios between the three axes of any given galaxy. Only two theoretical papers have so far attempted to model a specific galaxy, NGC 5128, in terms of a recent infall and a transient gas disk (Tubbs 1980; Simonson 1982). I suspect that until such evolutionary models are computed for many more galaxies, we may not reach our goal of determining the intrinsic shapes of ellipticals with confidence. 


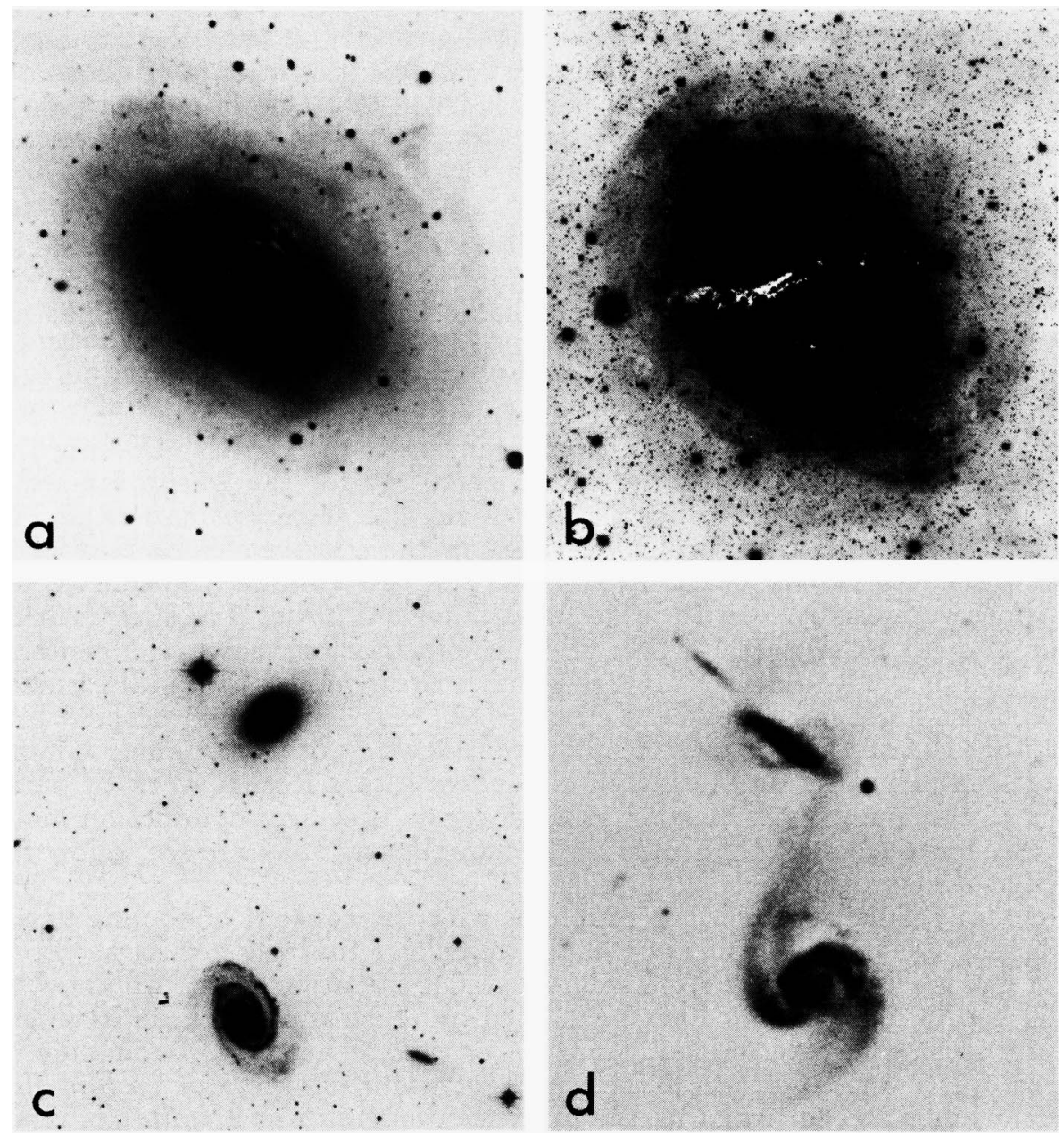

Figure 5. Accretion of disk material in ellipticals. (a) Ripples and dust in NGC 1316 (from Schweizer 1980); (b) ripples or "shells" in NGC 5128 (from Malin et al. 1983); (c) NGC 5363 and spiral neighbor NGC 5364; and (d) mass transfer between NGC 3808 and edge-on (S0?) companion (from Arp 1966).

\section{ATMOSPHERIC BALANCE}

There are two main questions concerning the atmospheric balance in ellipticals, and each question consists of various parts: 1) What are the sources and sinks of gas, and what is the net balance? 2) What are the main heating and cooling mechanisms, and why is there gas at three different temperatures? The answers to these questions are intertwined and, with one exception, difficult and not yet fully understood. In the brief space available here, I first address the one easy question and then mention a few points that seem important to me.

The relatively easy question concerns the sources of gas in ellipticals. There seem to be two of them. One source is the matter shed by evolving stars, including 
Miras, planetaries, novae, and supernovae. The total present mass-loss rate may be estimated from the mass difference between a turnoff star and an average white dwarf $\left(\Delta M \approx 0.3 M_{\odot}\right)$ and the theoretical evolution rate of $\mathrm{K}$ giants. The canonical number remains the mass-loss rate of $0.015 M_{\odot} \mathrm{yr}^{-1}\left(10^{9} L_{\odot}\right)^{-1}$ derived by Faber \& Gallagher (1976). For average giant ellipticals of $10^{10}-10^{11} L_{\odot}$, this rate amounts to $\sim 10^{9}-10^{10} M_{\odot} / 10^{10} \mathrm{yr}$. The second source of gas is infall by accretion or merging. I have estimated earlier from ripple statistics that an average giant $\mathrm{E}$ may have experienced 4 - 10 accretion events over a Hubble time (Schweizer 1983). Even if the average accretion event is only the proverbial "small gas-rich galaxy" that falls in, e.g., a Magellanic Cloud, the associated gas mass may be not much less than $10^{9} M_{\odot}$. The main point is, then, that $4-10 \mathrm{such}$ events can supply between a few times $10^{9} M_{\odot}$ and $10^{10} M_{\odot}$ of gas, or an amount quite comparable with all the gas shed by evolving stars.

What is the gas inflow, and is there a sink near the center? Cooling times for the gas are short near the center, of order $10^{6} \mathrm{yr}$ for the warm, ionized gas (Spitzer 1942) and $10^{8}-10^{9}$ yr for the hot X-ray gas (e.g., Forman et al. 1985; Canizares et al. 1986), though cooling times in the outer envelope may exceed a Hubble time. As Fabian discusses elsewhere in this volume, the short central cooling times supposedly lead to large mass inflows of order $1 M_{\odot} \mathrm{yr}^{-1}$ in field ellipticals and up to several $10^{2} M_{\odot} \mathrm{yr}^{-1}$ in galaxies located at the centers of clusters. Also in this volume, O'Connell reviews the evidence that star formation in such cooling flows may provide a sink for the gas. We should keep in mind, however, that direct observations of inflow motions do not exist. There is reason to be cautious in relying on calculated gas inflow rates: Recent work by various groups (e.g., Bertschinger \& Meiksin 1986) suggests that heat conduction may be significantly more efficient than previously thought and may reduce inflow rates by an order of magnitude.

Personally, I see some serious problems with the concept of cooling flows in ellipticals with gas disks. On the one hand, we believe that the hot X-ray coronae consist of gas shed by the stars of the galaxy. On the other hand, we know that the cooler gas in disks cannot have been shed by these stars because its angular momentum is generally decoupled from that of the stellar body. I conclude that the observed $H$ I and $H$ II gas disks cannot be manifestations of cooling flows. This conclusion is strengthened by the presence of significant amounts of dust in the same disks. Dust grains get destroyed rapidly in $10^{7} \mathrm{~K}$ gas and have lifetimes against sputtering of only $10^{6}-10^{7}$ yr (Draine \& Salpeter 1979). Therefore, the gas disks containing the dust cannot have condensed from the hot coronae. Yet if cooling flows of $\sim 1 M_{\odot} \mathrm{yr}^{-1}$ exist in these same coronae, funneling masses comparable to those of the disks in $10^{8}-10^{9} \mathrm{yr}$ toward the center, how come cloud-cloud collisions do not destroy the gas disks in similarly short times? To escape the problem of angular-momentum misalignment we could postulate that the hot coronae themselves consist largely of accreted gas, but this does still not solve the problem of the dust. Therefore, I remain skeptical of $\sim 1 M_{\odot} \mathrm{yr}^{-1}$ cooling flows as a general phenomenon in ellipticals.

What is the gas outflow, and is there a sink at infinity? In response to the apparent lack of gas in ellipticals observed in the late 1960's and early 1970's, Johnson \& Axford (1971) and Mathews \& Baker (1971) introduced the hypothesis of galactic winds powered by supernovae. In such a wind, fresh gas is continuously supplied by evolving stars. The energy released by cloud-cloud collisions occurring with velocities comparable to the random motions of the stars heats this gas to 
million-degree temperatures, but not enough to drive a wind. It is the combined energy input of many supernovae through their shockfronts that supposedly deposits enough extra heat to drive a hot wind and flush the gas out of the galaxy. The question before us today is whether this hypothesis of galactic winds is still necessary now that we observe about as much gas in X-ray coronae as is expected.

Already in 1979, Norman and Silk concluded that heating by supernovae should be important only during the relatively early phases of elliptical evolution with their presumedly violent star formation. They predicted that supernovae would not deliver sufficient energy to keep galactic winds blowing through the later, more quiescent phases of E evolution, especially not in massive galaxies (see also Larson 1974). Recent observations seem to have proven Norman and Silk right on two important counts: 1) Hot coronae have indeed been found to be common and especially pronounced in massive ellipticals; and 2) IRAS observations have opened our eyes to the fact that in colliding and merging galaxies the energy input into the gas can be prodigious. An interesting example is provided by NGC 3256, a pair of colliding spirals believed to be in the process of merging. Graham et al. (1984) point out that alone the supernovae associated with the starburst will dump one order of magnitude more energy into the gas than is required to blow it out of the combined potential well of the galaxies.

In conclusion, the answer to the question about gas outflow may be that galactic winds play an important role in ridding merging galaxies of their gas, and perhaps even in helping create some gas-free ellipticals. But these winds may then die down and leave the E's to rebuild their own atmospheres from both internal and external sources.

\section{CLOSING REMARKS}

How does our knowledge of dust and gas in ellipticals fit in with theories of elliptical formation and evolution? Instead of trying to give a general answer, let me return once more to one of my favorite ellipticals, NGC 5018 (Fig. 1), and comment on a few related issues, including some raised earlier at this Symposium.

First, note that NGC 5018 used to be a bona fide E. De Vaucouleurs classified it as an E3 in the RC2, and Sandage as an E4 in the Revised Shapley-Ames Catalog. Now, based on a new plate taken at Las Campanas, Sandage calls it an So because it has too much dust to be an $\mathrm{E}$ (private communication). Yet CCD photometry shows no trace of any disk. I believe it does not matter whether we call such galaxies E's or S0's; the story which they tell remains the same.

Second, it is easy to see from Figure 1 that brightness profiles extracted at different position angles across the nucleus will not show a perfect $r^{1 / 4}$ law. There will be deviations from such a law which cannot be blamed on faulty sky subtraction, but which reflect the presence of fine structure in the galaxy. These deviations will diminish if, as is now usual, mean brightness profiles are derived by some method of azimuthal averaging. Yet Figure 1 cautions us that we should not put too much emphasis on intercomparing such mean profiles. If we do, we may miss the most telling signatures such as ripples and tails.

Third, if we believe in Quinn's (1984) shell models and in tidal tails, NGC 5018 must have accreted some disk material - including stars, dust, and gas - not so long ago. The amount is surprising: From CCD photometry Fort et al. (1986) estimate that the extra luminous matter is $9 \%-18 \%$ of the total. Seitzer and I are finding similar amounts for several northern ellipticals with ripples. Even 
if perhaps above average, these amounts, together with the estimate of $4-10$ accretion events per Hubble time, suggest that a significant fraction of the visible matter in ellipticals may stem from a variety of foreign sources.

Dust and gas then seem to fit comfortably into a picture of elliptical formation and evolution based on mergers: Ellipticals may occasionally form from mergers of galaxies of nearly equal mass (Toomre \& Toomre 1972). Sporadically they continue accreting companions and matter from galaxies passing by. The gas and dust get stored temporarily in inclined disks, while the luminous matter is incorporated into the main bodies. Although there is evidence that these main bodies may be roughly triaxial, projection effects are unlikely to explain all isophotal twists. Some of the observed twists obviously reflect major recent perturbations, such as in NGC 5018, 1316, and 5128, and must correspond to intrinsic twists of the main axes. I believe the combined data suggest strongly that even in present-day ellipticals some growth and reshaping are still occurring.

I thank Halton C. Arp, William C. Forman, John A. Graham, and Jacqueline H. van Gorkom for their kind permission to reproduce some figures, and gratefully acknowledge partial support from the National Science Foundation through grant AST 83-18845.

\section{REFERENCES}

Arp, H.C.: 1966, Atlas of Peculiar Galaxies (Pasadena: California Institute of Technology).

Bertola, F., and Galletta, G.: 1978, Astrophys. J. 226, L115.

Bertola, F., Bettoni, D., Rusconi, L., and Sedmak, G.: 1984, Astron. J. 89, 356.

Bertola, F., Galletta, G., Zeilinger, W.W.: 1985, Astrophys. J. Lett. 292, L51.

Bertschinger, E., and Meiksin, A.: 1986, Astrophys. J. Lett. 306, L1.

Binney, J.: 1978, M.N.R.A.S. 183, 779.

Bottinelli, L., and Gouguenheim, L.: 1977, Astron. Astrophys. 54, 641.

Caldwell, N.: 1984a, Astrophys. J. 278, 96.

Caldwell, N.: 1984b, Publ.A.S.P. 96, 287.

Caldwell, N., Kirshner, R.P., and Richstone, D.O.: 1986, Astrophys. J. 305, 136.

Canizares, C.R., Donahue, M.E., Trinchieri, G., Stewart, G.C., and McGlynn, T.A.: 1986, Astrophys. J. 304, 312.

Carter, D., Jorden, P.R., Thorne, D.J., Wall, J.V., and Straede, J.C.: 1983, M.N.R.A.S. 205, 377 .

Davies, R.L., et al.: 1984, Bull. A.A.S. 16, 410.

Davies, R.L., and Illingworth, G.D.: 1986, Astrophys. J. 302, 234.

Demoulin-Ulrich, M.-H., Butcher, H.R., and Boksenberg, A.: 1984, Astrophys. J. $285,527$.

Draine, B.T., and Salpeter, E.E.: 1979, Astrophys. J. 231, 77.

Ebneter, K., and Balick, B.: 1985, Astron. J. 90, 183.

Faber, S.M., and Gallagher, J.S.: 1976, Astrophys. J. 204, 365.

Ford, H.C., and Butcher, H.: 1979, Astrophys. J. Suppl. 41, 147.

Forman, W., Schwarz, J., Jones, C., Liller, W., and Fabian, A.C.: 1979, Astrophys. J. Lett. 234, L27.

Forman, W., Jones, C., and Tucker, W.: 1985, Astrophys. J. 293, 102.

Fort, B.P., Prieur, J.-L., Carter, D., Meatheringham, S.J., and Vigroux, L.: 1986, Astrophys. J. 306, 110. 
Fosbury, R.A.E., Mebold, U., Goss, W.M., and Dopita, M.A.: 1978, M.N.R.A.S. $183,549$.

Gallagher, J.S., Knapp, G.R., Faber, S.M., and Balick, B.: 1977, Astrophys. J. $215,463$.

Gisler, G.R.: 1978, M.N.R.A.S. $183,633$.

Graham, J.A.: 1979, Astrophys. J. 232, 60.

Graham, J.R., Wright, G.S., Meikle, W.P.S., Joseph, R.D., and Bode, M.F.: 1984, Nature 310, 213.

Gunn, J.E.: 1979, in Active Galactic Nuclei, ed. C. Hazard and S. Mitton (Cambridge: Cambridge University Press), p. 213.

Hawarden, T.G., Elson, R.A.W., Longmore, A.J., Tritton, S.B., and Corwin, H.G., Jr.: 1981, M.N.R.A.S. 196, 747.

Heiligman, G., and Schwarzschild, M.: 1979, Astrophys. J. 233, 872.

Heisler, J., Merritt, D., and Schwarzschild, M.: 1982, Astrophys. J. 258, 490.

Hubble, E.: 1936, The Realm of the Nebulae (New York: Dover), p. 39.

Humason, M.L., Mayall, N.U., and Sandage, A.R.: 1956, Astron. J. 61, 97.

Johnson, H.E., and Axford, W.I.: 1971, Astrophys. J. 165, 381.

Kahn, F.D., and Woltjer, L.: 1959, Astrophys. J. 130, 705.

Knapp, G.R., Gallagher, J.S., and Faber, S.M.: 1978, Astron. J. 83, 139.

Knapp, G.R., Turner, E.L., and Cunniffe, P.E.: 1985, Astron. J. 90, 454.

Kumar, C.K., and Thonnard, N.: 1983, Astron. J. 88, 260.

Larson, R.B.: 1974, M.N.R.A.S. 169, 229.

Lauer, T.: 1985, M.N.R.A.S. 216, 429.

Malin, D.F., and Carter, D.: 1983, Astrophys. J. 274, 534.

Malin, D.F., Quinn, P.J., and Graham, J.A.: 1983, Astrophys. J. Lett. 272 , L5.

Mathews, W.G., and Baker, J.C.: 1971, Astrophys. J. 170, 241.

Mayall, N.U.: 1936, Publ. A.S.P. 48, 14.

Mayall, N.U.: 1939, Publ. A.S.P. 51, 282.

Merritt, D., and de Zeeuw, T.: 1983, Astrophys. J. Lett. 267, L19.

Minkowski, R., and Osterbrock, D.: 1959, Astrophys. J. 129, 583.

Norman, C., and Silk, J.: 1979, Astrophys. J. Lett. 233, L1.

Osterbrock, D.E.: 1960, Astrophys. J. 132, 325.

Phillips, M.M., Jenkins, C.R., Dopita, M.A., Sadler, E.M., and Binette, L.: 1986, Astron. J. 91, 1062.

Quinn, P.J.: 1984, Astrophys. J. 279, 596.

Rieke, G.H., and Lebofsky, M.J.: 1986, Astrophys. J. 304, 326.

Sadler, E.M., and Gerhard, O.E.: 1985, M.N.R.A.S. 214, 177.

Sandage, A.: 1957, Astrophys. J. 125, 422.

Schwarzschild, M.: 1979, Astrophys. J. 232, 236.

Schweizer, F.: 1980, Astrophys. J. 237, 303.

Schweizer, F.: 1983, in Internal Kinematics and Dynamics of Galaxies, IAU Symposium No. 100, ed. E. Athanassoula (Dordrecht: Reidel), p. 319.

Schweizer, F., and Ford, W.K., Jr.: 1985, in New Aspects of Galaxy Photometry, ed. J.-L. Nieto (Berlin: Springer), p. 145.

Sharples, R.M., Carter, D., Hawarden, T.G., and Longmore, A.J.: 1983, M.N. R.A.S. $202,37$.

Simonson, G.F.: 1982, Ph. D. thesis, Yale University.

Sparks, W.B., et al.: 1985, M.N.R.A.S. 217, 87.

Spitzer, L., Jr.: 1942, Astrophys. J. 95, 329.

Steiman-Cameron, T.Y., and Durisen, R.H.: 1982, Astrophys. J. Lett. 263, L51.

Steiman-Cameron, T.Y., and Durisen, R.H.: 1987, Astrophys. J., submitted. 
Tohline, J.E., and Durisen, R.H.: 1982, Astrophys. J. 257, 94.

Tohline, J.E., Simonson, G.F., and Caldwell, N.: 1982, Astrophys. J. 252, 92.

Toomre, A., and Toomre, J.: 1972, Astrophys. J. 178, 623.

Trinchieri, G., and Fabbiano, G.: 1985, Astrophys. J. 296, 447.

Tubbs, A.D.: 1980, Astrophys. J. 241, 969.

van Albada, T.S., Kotanyi, C.G., Schwarzschild, M.: 1982, M.N.R.A.S. 198, 303.

van Gorkom, J.H., Knapp, G.R., Raimond, E., Faber, S.M., and Gallagher, J.S.: 1986, Astron. J. 91, 791.

Wilkinson, A., Sharples, R.M., Fosbury, R.A.E., and Wallace, P.T.: 1986, M.N. R.A.S. 218, 297.

\section{DISCUSSION}

King: In the picture of ellipticals formed by merging of spirals, there is the problem that ellipticals have more globular clusters than spirals. How do you reconcile this?

Schweizer: This problem is not really serious in my view, at least not yet. It is often cited as an objection to the formation of ellipticals through mergers of spirals, yet it seems to be based on two unproven, and possibly wrong, promises: (i) All globular clusters in any galaxy are as old as those in the Milky Way seem to be; and (ii) the number of globular clusters in a merger remnant is the sum of the globular clusters in the pre-merger galaxies.

Concerning the first premise, we know that young and intermediate-age globular clusters exist in the Magellanic Clouds, M33, and M31. It just happens that our Milky Way doesn't seem to possess any. So at least three or four out of five nearby galaxies do know how to make globular clusters long after the Big Bang.

Concerning the second premise, we still know little about the details of star formation in mergers of gas-rich galaxies. Yet we do know that such "super starburst" galaxies as Arp 220 and NGC 6240 produce vast anounts of molecular gas (Joseph, Wright \& Wade, 1984, Nature, 311, 132; Rieke, Cutri, Black, Kailey, McAlary, Lebofsky \& Elston, 1985, Astrophys. J., 290, 116) and achieve very high efficiencies of star formation. Both objects seem to be ongoing mergers of about equal-sized disk galaxies. It seems unreasonable to me that both should have dense molecular clouds and form stars at prodigious rates, yet they would not also produce globular clusters. What better environment is there to produce massive clusters than the highly crunched gas in such systems? If this view is correct and ellipticals arise from disk mergers, one would certainly expect the specific globular cluster frequency to be higher in ellipticals than in the pre-merger galaxies.

Norman: You need $\sim 10$ gas-rich dwarfs per Hubble time to give the gas in ellipticals. Where are these dwarfs or, more precisely, where have they been? A current gas-rich dwarf density of 10 per $L^{*}$ galaxy is not observed. Were they eaten, are they found in interactions, or have they lost their gas and become very low surface brightness objects?

Schweizer: My statement was only that there seem to have been 4-10 accretion events per average giant elliptical over the age of the Universe. This accretion rate is estimated from the number of ellipticals with ripples (about 50\%) that I detected 
in a CTIO 4-meter survey some years ago, and from estimated ripple lifetimes $(\sim 2$ Gyr; see IAU Symp. 100, p. 319, 1983). I believe that the majority of these events were not mergers of small, gas-rich galaxies, but rather less glamorous transfers of mass from the outskirts of neighboring disk galaxies. The reason is that the cross section for mass transfer must be of order $10-10^{2}$ times larger than the cross section for mergers. It takes only a near collision to induce mass transfer, but a very nearly central hit to start a merger. And as we well know, the outskirts of disk galaxies are, on average, quite gas rich. I would, therefore, conclude that foreign gas and stars in ellipticals probably stem from a variety of sources, and that some of the neighbor galaxies that donated material in the past will do it again in the future. Another way to say this is that galaxies seem to be notoriously promiscuous!

Toomre: [Question addressed to Tjeerd van Albada.] After hearing Schweizer mention with some skepticism the test proposed by yourself and Schwarzschild and Kotanyi about the sense of warp in a postulated tumbling triaxial Cen $\mathbf{A}$ potential, do you yourself agree now that your test has been flunked in that famous example?

van Albada: I will let Martin Schwarzschild answer this.

Schwarzschild: Yes, Alar Toomre, we have flunked the test. But I would only retreat half way. I now think that the truth in this matter-as in so many caseslies between the extreme pictures. The outer parts of the dust ring in Cen $\mathbf{A}$ and other systems, with their twists and rugged appearance, seem not yet settled on stable orbits but the inner parts, for which the viscous settling time is shorter, have it seems managed to settle on stable orbits. Hence, triaxiality is still needed for Cen $\mathbf{A}$ to provide a stable orbit for the inner part of the dust ring but fast figure rotation-in a prescribed sense-is not anymore needed to explain the twists of the outer parts which we now assume are relics of the merger geometry. 


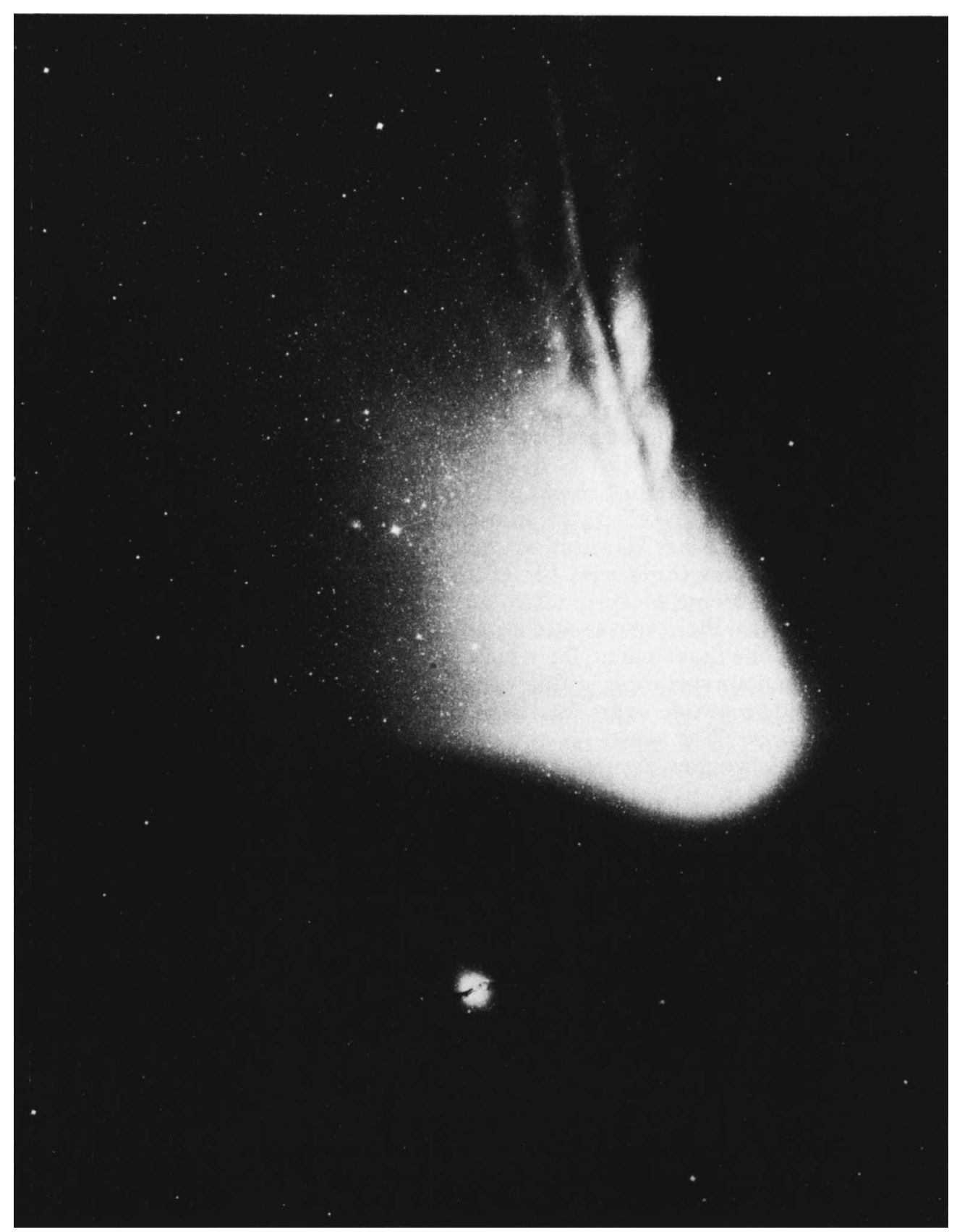

Centaurus $A$, with comet $P /$ Halley, as presented by Schweizer. The $8^{m}$ exposure on a $103 a O$ plate was taken on April 15, 1986, by $A$. Gomez with the Curtis Schmidt telescope at Cerro Tololo (National Optical Astronomy Observatories). 